\title{
JPE 15-4-2
}

\section{A Controllable LCL-T Resonant AC/DC Converter for High Frequency Power Distribution Systems}

\author{
Jun Zeng ${ }^{*}$ Xuesheng $\mathrm{Li}^{*}$, and Junfeng $\mathrm{Liu}^{\dagger}$ \\ *School of Electric Power, South China University of Technology, Guangzhou, China \\ ${ }^{\dagger}$ School of Automation Science and Engineering, South China University of Technology, Guangzhou, China
}

\begin{abstract}
High frequency alternating current (HFAC) has been widely used in a wide range of power distribution systems (PDS) due to its superior performance. A high frequency AC/DC converter plays the role of converting HFAC voltage to DC voltage. In this paper, a new $L C L-T$ resonant AC/DC converter has been proposed, and an easier control method based on input voltage comparison is presented, without the complicated calculation of the zero-crossing point. Both a low distortion and near-to-unity power factor can be achieved by the proposed resonant converter and control strategy. The operational principle and steady-state analysis are given for the proposed resonant converter. A simulation model and experimental prototype are implemented with an operation frequency of $25 \mathrm{kHz}$ and a rated power of $20 \mathrm{~W}$. The simulation and experimental results verify the accuracy of the analysis and the excellent performance of the proposed topology.
\end{abstract}

Key words: AC/DC converter, Controllable resonant converter, High frequency alternating current (HFAC)

\section{INTRODUCTION}

In consideration of the outstanding performance involving fast dynamic response, high power density and flexible voltage grade, high frequency AC (HFAC) plays an important role in a wide range of power distribution systems (PDS), such as spacecraft applications, telecommunication systems, computer and electronic commercial systems, automotive applications and Micro-grids [1]-[14]. Traditionally, there are two types of power conversion in HFAC PDS: 1) conversion from DC to HFAC [8]-[14], and 2) conversion from HFAC to DC. When compared with DC/HFAC inverters, the following objectives are significant for HFAC/DC conversion: 1) lighter weight and higher power density; 2) controlled DC output over a wide range; 3) lower electromagnetic interference (EMI); 4) higher reliability; 5) lower distortion, near-sinusoidal input current and close-to-unity power factor. Consequently, a number of studies have been conducted from the circuit topology to the control scheme.

It has been shown that using passive components is more effective than the active power factor correction (PFC)

Manuscript received Nov. 24, 2014; accepted Mar. 10, 2015

Recommended for publication Associate Editor by Honnyong Cha.

${ }^{\dagger}$ Corresponding Author: aujfliu@scut.edu.cn

Tel: +86-20-87114828, South China University of Technology

*School of Electric Power, South China University of Technology, China techniques for HFAC/DC converter for achieving the objectives of a high input power factor and high efficiency [15]. When compared with valley fill and charging pump circuits, the resonant converter is a better PFC choice [16], [17]. A number of approaches for the design of resonant AC/DC converters have been proposed in [18]-[23]. A resonant rectifier based on thyristor switches has been proposed with an $L C-T$ resonant network and phase shift modulation (PSM). A close-to-unity power factor and low input harmonics have been achieved in [18], [19]. However, the implementation of PSM is complicated for thyristors. Phase density modulation (PDM) has been proposed for AC/DC converter formed by a resonant network, a bidirectional shunt switch, a transformer synchronous rectifiers and an output filter [20], [21]. However, more components and a complicated control limit its application. A resonant topology for an AC/DC converter has been reported in [24], [25], which is constituted by an $L C L-T$ resonant network and a half-bridge switch. If the $L C L-T$ resonant tank operates at the resonant frequency, the resonant converter behaves like a current source [26]-[29]. Moreover, it is inherently output short-circuit proof due to the existence of clamped diodes [25]. The $L C L-T$ resonant converter satisfies the need for a constant current characteristic and close-to-unity power factor, but it is incapable of providing a controllable output. The conventional control of output voltage is to 
regulate the front-end converter. However, this requires more power components and has a high cost [27]. In order to simplify the interleaved circuit, this paper presents a controllable converter topology based on a $L C L-T$ resonant tank and a bidirectional ac switch. In addition, an easier control scheme is presented for the proposed topology without the usual zero-crossing point detection of resonant current.

A wide scope of output regulation can be achieved by the proposed converter and control scheme with a low distortion input current and a close-to-unity input power factor. This paper is organized as follows. A detailed description of the proposed $L C L-T$ resonant converter and its operating principle are given in Section II. A steady-state analysis is performed in Section III with a performance discussion, including the output voltage control, input power factor, and total harmonic distortion (THD) of the input current. Simulation results of the $L C L-T$ resonant AC/DC converter are presented in Section IV to verify the analysis. Section $\mathrm{V}$ provides experimental results and Section VI summarizes the conclusion drawn from the investigation.

\section{CIRCUIT DESCRIPTION AND OPERATING PRINCIPLE}

The proposed AC/DC converter is shown in Fig. 1. It is formed by an $L C L-T$ resonant network, a bidirectional ac switch and a full-bridge rectifier. The $L C L-T$ resonant network is comprised by a capacitor $C$ and two inductors $L_{1}$ and $L_{2}$, where the inductance of $L_{1}$ is close to $L_{2}$. The bidirectional ac switch is comprised by two MOSFET switches $Q_{1}$ and $Q_{2}$ connected back to back. The backend rectifier is constructed by full-bridge diodes $D_{1}, D_{2}, D_{3}$, and $D_{4}$ and an output filter capacitor $C_{o}$. The input is a HFAC voltage $v_{s}$, and $R_{o}$ is the load resistance.

Traditionally, high frequency sinusoidal voltage $v_{s}$ is derived from a high frequency AC bus. An LCL-T resonant circuit converts the HFAC voltage into a current source when the input frequency is equal to the resonant frequency. The impedance of the $L C L-T$ resonant tank to the fundamental component is significantly less than the impedance to high order harmonics, which effectively reduces the THD of the input current. Therefore, the harmonics distortion of the input side is negligible under the given load conditions. Meanwhile, the fundamental component of the input current and the input voltage are nearly in the same phase if the inductance of $L_{1}$ is close to $L_{2}$ [25]. Therefore, a close-to-unity power factor can be achieved.

When the resonant components $L_{1}, C$ and $L_{2}$ are tuned to the input frequency, the resonant tank is viewed as an ideal current source without an internal impedance due to a high impedance to the harmonic components [25], [26]. With the predefined resonant components and input voltage $v_{s}$, the resonant current passing through the inductor $L_{2}$ has a constant amplitude. The

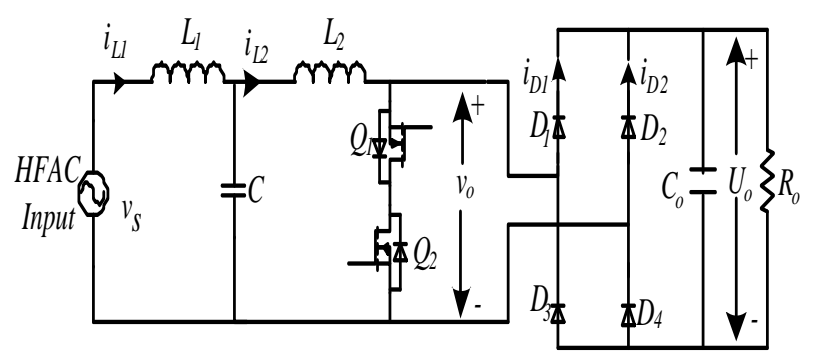

Fig. 1 The proposed AC/DC converter.

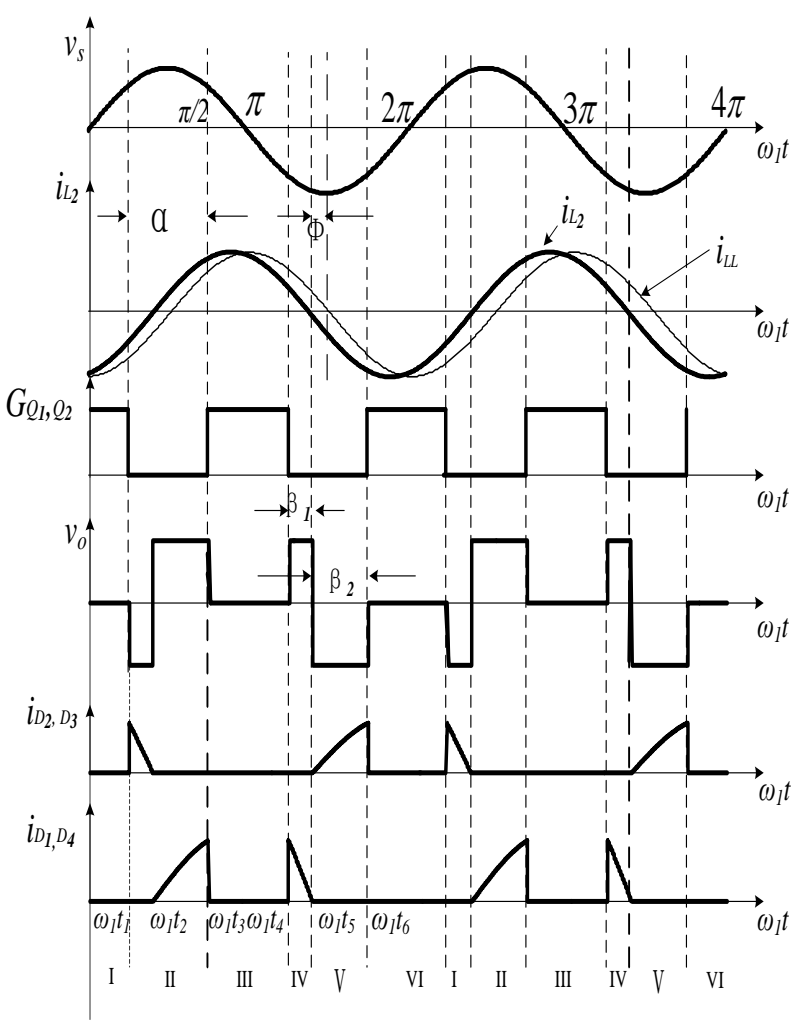

Fig. 2 The waveforms of the proposed AC/DC converter

bidirectional ac switch is adopted to control the amount of bidirectional current between the resonant tank and the rectifier. The full-bridge rectifier converts the controllable bidirectional current to a unidirectional current for the load. In the existing control of resonant AC/DC converters, it is necessary to calculate the angle between the input voltage and the current pouring into the rectifier. When compared to the complicated current monitor, an easier control scheme based on an input voltage comparison is presented and analyzed in this paper. By means of regulating the conducting angle of $Q_{1}$ and $Q_{2}$, the amount of current feeding the rectifier is adjusted. As a result, the output voltage and output current are controllable. A filter capacitor $C_{o}$ is used to filter the output ripples and to provide a constant DC voltage to the load.

The operating waveforms of the proposed AC/DC topology are demonstrated in Fig. 2. $i_{L 2}$ is the current passing through $L_{2}$, and $i_{L L}$ is the reference waveform with a phase angle lagging 


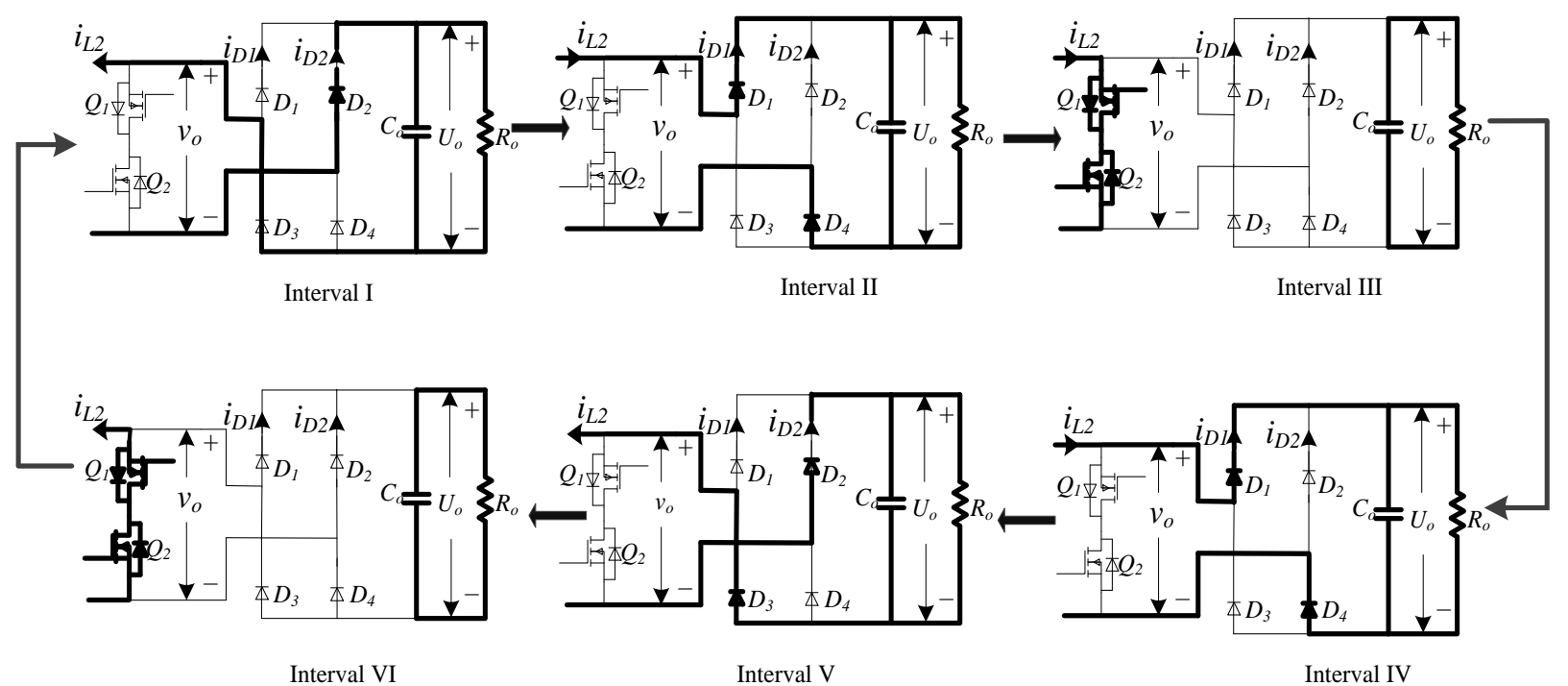

Fig. 3. Current path in switches $Q_{1}, Q_{2}$ and diodes $D_{1}, D_{2}, D_{3}, D_{4}$ in six intervals in one operating cycle.

$90^{\circ}$ behind the input voltage. The phase difference between the resonant current $i_{L 2}$ and $i_{L L}$ is $\Phi . G_{Q 1, Q 2}$ is the driving signals of the switches $Q_{1}$ and $Q_{2} . v_{o}$ is the voltage across the MOSFET switches. $i_{D 1, D 3}$ is the current passing through the diodes $D_{1}$, $D_{3}$; while $i_{D 2, D 4}$ is the current passing through the diodes $D_{2}, D_{4} . \beta_{1}$ is the conducting angle of $D_{1}, D_{3}$; and $\beta_{2}$ is the conducting angle of $D_{2}, D_{4}$ during an half cycle of the input voltage. $\alpha$ is the charging angle of the output capacitor $C_{o}$. The driving signals are symmetrical with respect to the zero point of the input voltage. Therefore, it is unnecessary to calculate the angle between the input voltage and the current pouring into the full-bridge rectifier. Each operating cycle contains six intervals. The current path of the different states is shown in Fig. 3, and the operational analysis is explained as follows.

\section{Interval I}

Interval I begins at $\omega_{1} t_{1}=(\pi-\alpha) / 2 . Q_{1}$ and $Q_{2}$ are turned off. The resonant current $i_{L 2}$ passes through the diodes $D_{2}$ and $D_{3}$ to charge the output capacitor $C_{o}$. Since the ripple is filtered by the capacitor $C_{o}$, the output voltage is $U_{o}$ with a constant value. The voltage $v_{o}$ over the switches $Q_{1}$ and $Q_{2}$ is equal to $-U_{o}$ in this interval.

Interval II

Interval II begins at $\omega_{1} t_{2}=\pi / 2-\Phi$. Since the direction of $i_{L 2}$ has reversed, the resonant current $i_{L 2}$ passes through the diodes $D_{1}$ and $D_{4}$ to charge the capacitor $C_{o}$. The voltage $v_{o}$ is equal to $U_{o}$ in this interval.

\section{Interval III}

Interval III begins at $\omega_{1} t_{3}=(\pi+\alpha) / 2 . Q_{1}$ and $Q_{2}$ are turned on. The resonant current $i_{L 2}$ passes through $Q_{1}$ and $Q_{2}$. The charging process of the capacitor $C_{o}$ is suspended. $C_{o}$ feeds the load and the voltage $v_{o}$ is equal to zero in this interval.

Interval IV

Interval IV begins at $\omega_{1} t_{4}=(3 \pi-\alpha) / 2 . Q_{1}$ and $Q_{2}$ are turned off. The resonant current $i_{L 2}$ passes through the diodes $D_{1}$ and
$D_{4}$ to charge the output capacitor $C_{o}$. The voltage $v_{o}$ is equal to $U_{o}$ in this interval.

\section{Interval $V$}

Interval $\mathrm{V}$ begins at $\omega_{1} t_{5}=3 \pi / 2-\Phi$. Since the direction of $i_{L 2}$ has reversed, the resonant current $i_{L 2}$ passes through the diodes $D_{2}$ and $D_{3}$ to charge the capacitor $C_{o}$. The voltage $v_{o}$ is equal to $-U_{o}$ in this interval.

\section{Interval VI}

Interval VI begins at $\omega_{1} t_{6}=(3 \pi+\alpha) / 2 . Q_{1}$ and $Q_{2}$ are turned on. The resonant current $i_{L 2}$ passes though $Q_{1}$ and $Q_{2}$. The charging process of the capacitor $C_{o}$ is suspended. $C_{o}$ feeds the load and $v_{o}$ is equal to zero in this interval.

After interval VI, a new cycle begins and the same operating principle is performed again. In order to make the output stable, the energy stored in the resonant network is regulated by $Q_{1}$ and $Q_{2}$ in each operating cycle.

\section{STEADY-STATE ANALYSIS}

Before the steady-state analysis is conducted, some assumptions are made: 1 ) all of the components and devices are ideal; 2) the input voltage has a constant amplitude with a fixed frequency; 3) the output DC voltage is ripple free and the output voltage is constant.

The converter can be equivalent to a circuit with two sources as shown in Fig. 4(a). One is the input voltage source $v_{s}$, and the other is the equivalent voltage source of the output rectifier $v_{o}$. Based on the superposition theorem, the equivalent circuit shown in Fig. 4(a) can be decomposed into two parts. The two parts shown in Fig. 4(b) and Fig. 4(c) can be calculated and analyzed separately.

Since the input voltage $v_{s}$ is ideal and without any harmonic components, the input voltage can be expressed as below.

$$
v_{s}=\sqrt{2} V_{s} \sin \left(\omega_{1} t\right)
$$




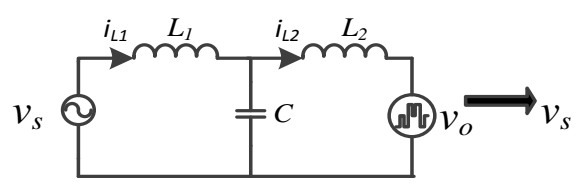

(a)

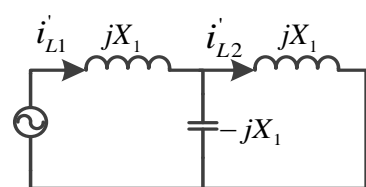

(b)

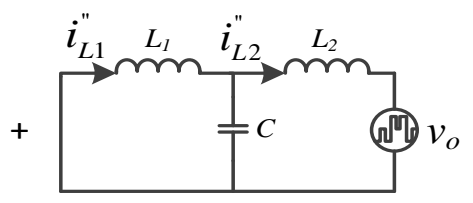

(c)

Fig. 4. Equivalent circuit of the controllable LCL-T resonant converter.

where, $V_{s}$ is the root mean square value (RMS) of the input voltage $v_{s}$.

Due to the ideal input voltage, the following relations of the current can be derived from Fig. 4(b).

$$
\begin{gathered}
i_{L_{1}}^{\prime}=0 \\
i_{L_{2}}^{\prime}=-\sqrt{2} \frac{V_{s}}{X_{1}} \cos \left(\omega_{1} t\right)
\end{gathered}
$$

where, $X_{1}$ is the fundamental impedance of the inductor $L_{1}$, and $X_{1}=\omega_{1} L_{1}=\omega_{1} L_{2}=1 / \omega_{1} C$. $i{ }^{\prime}{ }_{L 1}$ and $i{ }^{\prime}{ }_{L 2}$ are also sinusoidal waveforms without any high order harmonics.

The voltage $v_{o}$ can be expressed by the Fourier series as below.

$$
\begin{aligned}
v_{0}= & \sum_{n=1}^{\infty}\left\{\left(\cos \frac{n \alpha}{2}-\cos n \phi\right) \cdot \cos n \omega_{1} \mathrm{t}+\sin n \phi \cdot \sin n \omega_{1} \mathrm{t}\right\} . \\
& \frac{4 U_{o}}{n \pi} \cdot \sin \frac{n \pi}{2} \\
= & \sum_{n=1}^{\infty} \frac{4 U_{o}}{n \pi} \cdot \sin \frac{n \pi}{2} \cdot \sqrt{A_{n}^{2}+B_{n}^{2}} \cdot \cos \left(n \omega_{1} \mathrm{t}-\theta_{n}\right)
\end{aligned}
$$

where, $U_{o}$ is the output voltage, and $A_{n}=\cos (n \alpha / 2)-\cos (n \Phi)$, $B_{n}=\sin (n \Phi)$, and $\theta_{n}=\arctan \left(B_{n} / A_{n}\right)$. The circuit analysis is performed by a series of harmonics.

The currents of $i_{L 1}$ and $i_{L 2}{ }$ can be derived from Fig.4(c).

$$
\begin{aligned}
& i_{L_{1}}^{\prime \prime}=\sum_{n=1}^{\infty} \frac{4 U_{o}}{n\left(n^{3}-2 n\right) \pi X_{1}} \cdot \sin \frac{n \pi}{2} \cdot \sqrt{A_{n}^{2}+B_{n}^{2}} \cdot \sin \left(\mathrm{n} \omega_{1} \mathrm{t}-\theta_{n}\right) \\
& i_{L_{2}}^{\prime \prime}=\sum_{n=1}^{\infty}-\frac{4 U_{o}\left(n^{2}-1\right)}{n\left(n^{3}-2 n\right) \pi X_{1}} \cdot \sin \left(\frac{n \pi}{2}\right) \cdot \sqrt{A_{n}^{2}+B_{n}^{2}} \cdot \sin \left(\mathrm{n} \omega_{1} \mathrm{t}-\theta_{n}\right)
\end{aligned}
$$

\section{A. Determination of the Phase Difference Angle $\Phi$}

Furthermore, the total input current $i_{L 1}$ can be derived by (2) and (5).

$$
\begin{aligned}
i_{L_{1}} & =i_{L_{1}}^{\prime}+i_{L_{1}}^{\prime \prime} \\
& =0+\sum_{n=1}^{\infty} \frac{4 U_{o}}{n\left(n^{3}-2 n\right) \pi X_{1}} \cdot \sin \left(\frac{n \pi}{2}\right) \cdot \sqrt{A_{n}^{2}+B_{n}^{2}} \cdot \sin \left(\mathrm{n} \omega_{1} \mathrm{t}-\theta_{n}\right) \\
& =\sum_{n=1}^{\infty} \frac{4 U_{o}}{n\left(n^{3}-2 n\right) \pi X_{1}} \cdot \sin \left(\frac{n \pi}{2}\right) \cdot \sqrt{A_{n}^{2}+B_{n}^{2}} \cdot \sin \left(\mathrm{n} \omega_{1} \mathrm{t}-\theta_{n}\right)
\end{aligned}
$$

The fundamental component of the input current is:

$$
\begin{aligned}
i_{L_{1} \text { f fundamental }}= & -\frac{4 U_{o}}{\pi X_{1}} \cdot \sqrt{A_{1}^{2}+B_{1}^{2}} \cdot \sin \left(\omega_{1} \mathrm{t}-\theta_{1}\right) \\
= & \frac{4 U_{o}}{\pi X_{1}} \cdot \sqrt{\left(\cos \frac{\alpha}{2}-\cos \Phi\right)^{2}+(\sin \Phi)^{2}} \\
& \cdot \sin \left(\omega_{1} \mathrm{t}+\pi-\theta_{1}\right)
\end{aligned}
$$

The angle between the input fundamental current $i_{\text {L1.fundamental }}$ and the input voltage $v_{s}$ is $\pi-\theta_{1}$. Furthermore, the input power can be derived as below.

$$
P_{\text {in }}=V_{s} \cdot \frac{2 \sqrt{2} U_{o}}{\pi X_{1}} \cdot \sqrt{\left(\cos \frac{\alpha}{2}-\cos \Phi\right)^{2}+(\sin \Phi)^{2}} \cdot \cos \left(\pi-\theta_{1}\right)
$$

The output power is calculated from the output voltage and the load. The expression of the output power $P_{o}$ is given as below.

$$
P_{o}=U_{o}^{2} / R_{o}
$$

where, $R_{o}$ is the load resistance.

Due to the assumption that all of the components and devices are ideal and without losses, the input power $P_{\text {in }}$ is equal to the output power $P_{o}$. The following expression can be derived from (9) and (10).

$$
\frac{U_{o}}{V_{s}}=\frac{2 \sqrt{2} U_{o}}{\pi X_{1}} \cdot \sqrt{\left(\cos \frac{\alpha}{2}-\cos \Phi\right)^{2}+(\sin \Phi)^{2}} \cdot \cos \left(\pi-\theta_{1}\right)
$$

Similarly, the resonant current $i_{L 2}$ can be derived from (3) and (6).

$$
\begin{aligned}
i_{L_{2}}= & i_{L_{2}}^{\prime}+i_{L_{2}}^{\prime \prime} \\
= & -\sqrt{2} \frac{V_{s}}{X_{1}} \cos \omega_{1} \mathrm{t} \\
& +\sum_{n=1}^{\infty}-\frac{4 U_{o}\left(n^{2}-1\right)}{n\left(n^{3}-2 n\right) \pi X_{1}} \cdot \sin \frac{n \pi}{2} \cdot \sqrt{A_{n}^{2}+B_{n}^{2}} \cdot \sin \left(n \omega_{1} \mathrm{t}-\theta_{n}\right)
\end{aligned}
$$

It can be found from equation (12) that $i_{L 2}$ can be regulated by the input voltage $v_{s}$ and the inductance of $L_{2}$. Since a larger $i_{L 2}$ leads to more circulating current and reactive power, a suitable selection of $L_{2}$ is essential to cut down $i_{L 2}$.

As shown in Fig.2, $i_{L 2}$ reaches the zero point at the position $\omega_{1} t=(\pi / 2-\Phi)$. Therefore, the following equation is satisfied.

$$
\begin{aligned}
& \left(\sum_{n=1}^{\infty}-\frac{4 U_{o}\left(n^{2}-1\right)}{n\left(n^{3}-2 n\right) \pi X_{1}} \sin \frac{n \pi}{2} \cdot \sqrt{A_{n}^{2}+B_{n}^{2}} \cdot \sin \left[\mathrm{n}\left(\frac{\pi}{2}-\Phi\right)-\theta_{n}\right]\right) \\
& -\sqrt{2} \frac{V_{s}}{X_{1}} \cos \left(\frac{\pi}{2}-\Phi\right)=0
\end{aligned}
$$

By putting (11) into (13), the solution of equation (13) can be found. At a given $\alpha$ varying from $90^{\circ}$ to $180^{\circ}$, the solution is to find the zero point of a function of $\Phi$. As a result:

$$
\begin{aligned}
\left.f(\Phi)\right|_{\alpha \in\left(90^{\circ}, 180^{\circ}\right)} & -\sin \Phi+K \cdot\left(\cos \frac{\alpha}{2}-\cos \Phi\right) \\
& \cdot \sum_{n=1}^{\infty} \sin ^{2}\left(\frac{n \pi}{2}\right) \cdot \frac{n^{2}-1}{n\left(n^{3}-2 n\right)} \cdot\left[\cos n \Phi \cdot \cos \frac{\alpha}{2}-1\right]
\end{aligned}
$$

where, $K=8 R_{o} / \pi^{2} X_{1}$.

The relation curves of $\Phi$ to the control angle $\alpha$ with different value of $K$ are shown in Fig. 5(a). If the parameter $K$ is 


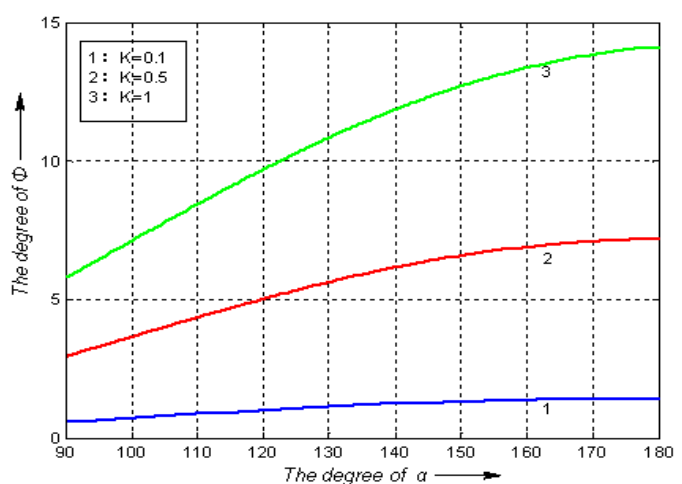

(a)

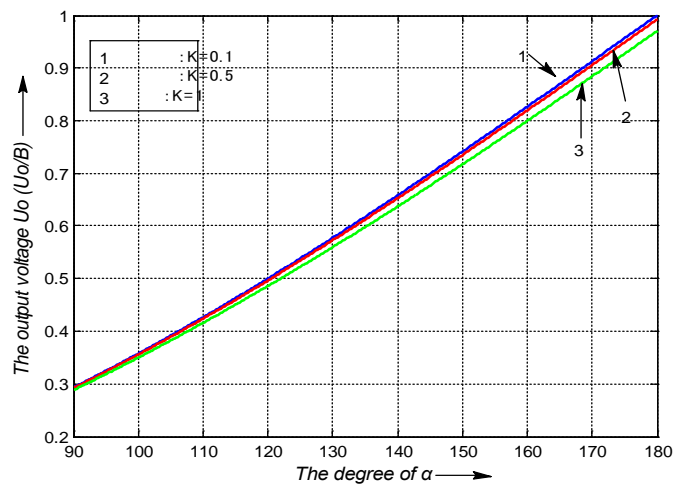

(b)

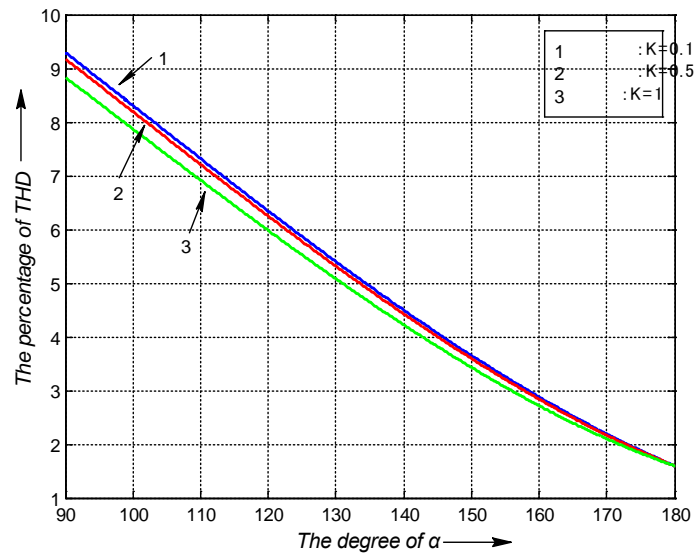

(c)

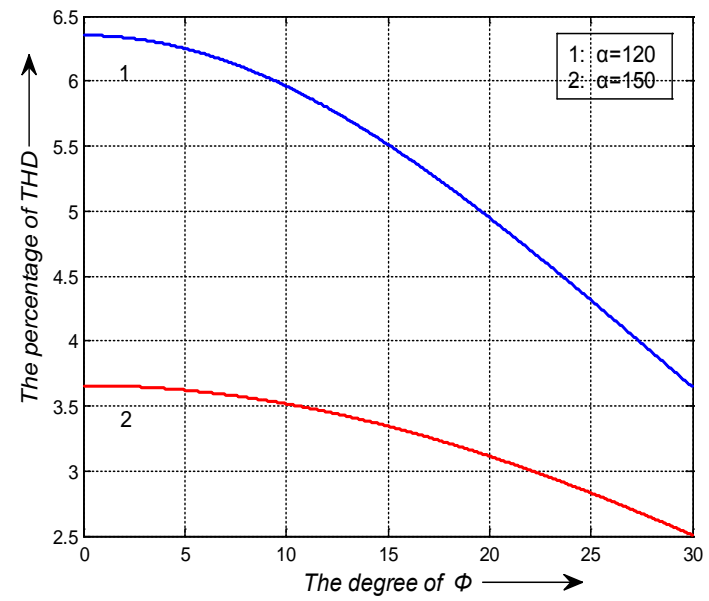

(d)

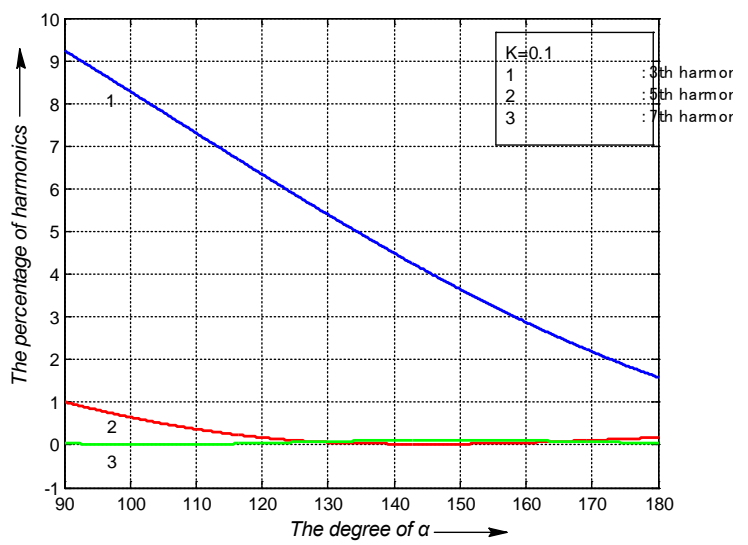

(e)

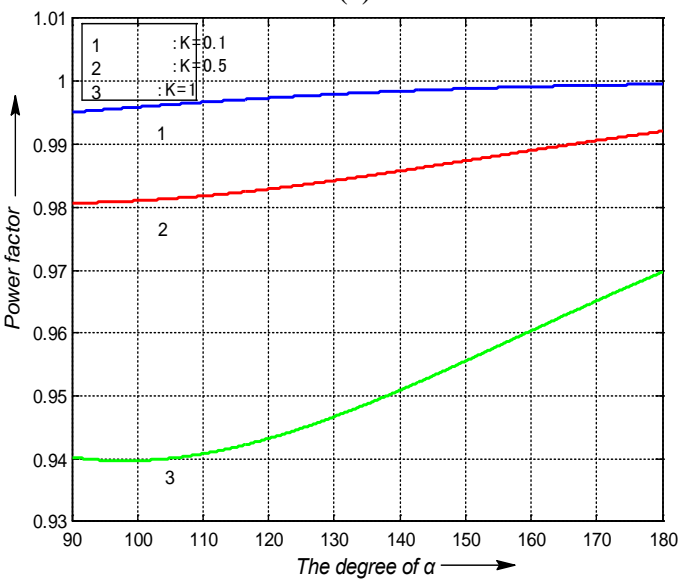

(f)

Fig. 5. (a) The relation curves of angle $\Phi$ to control angle $\alpha$ with different $K$. (b) The relation curves of voltage $U o / B$ to control angle $\alpha$ at different $K$. (c) The relation curves of THD to control angle $\alpha$ with different $K$. (d) The relation curves of THD to $\Phi$ with different $\alpha$. (e) The percentage of third, fifth, seventh harmonic of the input current as a function of control angle $\alpha$ $(K=0.1)$. (f) The relation curves of power factor to control angle $\alpha$ with different $K$.

predefined, the angle $\Phi$ increases as the control angle $\alpha$ increases. The angle $\Phi$ increases with an increase of $K$. The maximum $\Phi$ is close to $15^{\circ}$ when $K$ is 1 ; while the minimum $\Phi$ is close to $0^{\circ}$ when $K$ is 0.1 .

\section{B. Output Voltage $U_{o}$}

The output voltage $U_{o}$ can be derived from (11).

$$
U_{o}=B \cdot\left(\cos \phi-\cos \frac{\alpha}{2}\right)
$$

where, $B=2 \sqrt{2} V_{s} R_{o} / \pi X_{1}$.

$\alpha$ is the conduction angle of the switches $Q_{1}$ and $Q_{2}$. It is adjusted to achieve a desired output. $K$ is determined by the resonant components and load condition. The relation curves of the output voltage $U_{o} / B$ to control the angle $\alpha$ are shown in Fig. 5(b). It can be observed that the output voltage $U_{o}$ increases as $\alpha$ grows from $90^{\circ}$ to $180^{\circ}$. The output voltage $U_{o}$ can be regulated in a wide range from $29.28 \%$ to $99.97 \%$ when $K=0.1$. Meanwhile, the range is from $29.16 \%$ to $99.22 \%$ when $K=0.5$, 


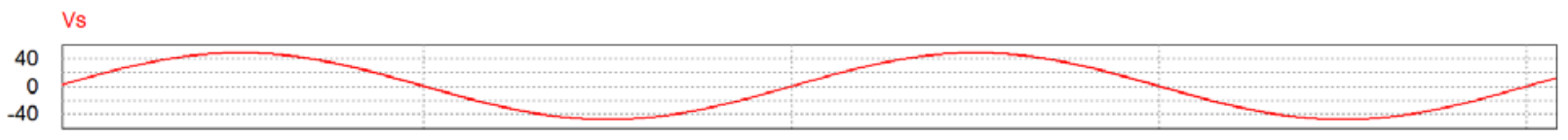

iL1

(a)
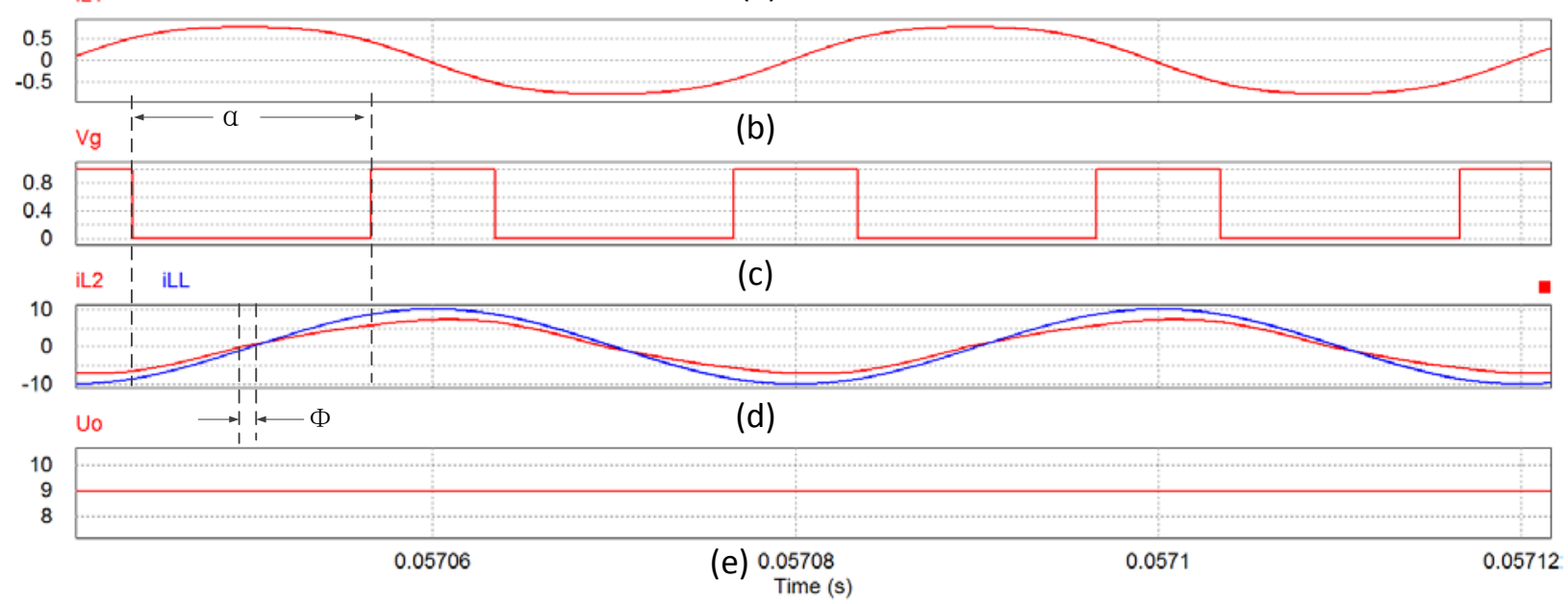

Fig. 6. Simulation waveforms of the proposed AC/DC converter, operation frequency is $25 \mathrm{kHz}$, output voltage is $9 \mathrm{~V}$.

and it is from $28.78 \%$ to $96.98 \%$ when $K=1$. The variation scope decreases slowly with the increase of $K$.

\section{THD of the Input Current}

The THD of the input current is defined as below.

$$
T H D=\frac{\sqrt{\sum_{n=2}^{\infty} I_{n}^{2}}}{I_{1}} \cdot 100 \%
$$

where, $I_{1}$ is the RMS of the fundamental current, and $I_{n}$ is the RMS of the $n^{\text {th }}$ harmonic current. The THD of the input current $i_{L 1}$ can be derived from (7) and (8) as below.

$$
\begin{aligned}
T H D & =\frac{\sqrt{\sum_{n=2}^{\infty}\left\{\frac{4 U_{o}}{\sqrt{2} \cdot n\left(n^{3}-2 n\right) \pi X_{1}} \cdot \sin \left(\frac{n \pi}{2}\right) \cdot \sqrt{A_{n}^{2}+B_{n}^{2}}\right\}^{2}}}{\frac{4 U_{o}}{\sqrt{2} \cdot \pi X_{1}} \cdot \sqrt{A_{1}^{2}+B_{1}^{2}}} \\
& =\frac{\sqrt{\sum_{n=2}^{\infty}\left\{\frac{\left[\cos \left(\frac{n \alpha}{2}\right)-\cos (n \phi)\right]^{2}+\sin ^{2}(n \phi)}{n\left(n^{3}-2 n\right)}\right\}}}{\sqrt{\left[\cos \left(\frac{\alpha}{2}\right)-\cos (\phi)\right]^{2}+\sin ^{2}(\phi)}}
\end{aligned}
$$

The relation curves of the THD to control the angle $\alpha$ with different values of $K$ are shown in Fig. 5(c). It can be found that the THD decreases with an increase of the angle $\alpha$. When $K$ increases, the THD decreases slightly. Fig. 5(d) shows the relation curves of the THD to the angle $\Phi$ with different values of $\alpha$. When the angle $\alpha$ is constant, the denominator of the THD in (17) decreases as the angle $\Phi$ decreases. This is proportional to the RMS of the fundamental current. Therefore, the THD decreases as the angle $\Phi$ increases. Fig. 5(e) shows the percentage of the third, fifth, seventh and ninth order harmonics of the input current as a function of the control angle $\alpha$ at $K=0.1$. It can be found that the third harmonic is the dominant harmonic and that the percentages of the other harmonics are low relatively.

\section{Power Factor}

The power factor is defined as below.

$$
P F=\frac{\cos \theta}{\sqrt{1+T H D^{2}}}
$$

where, $\theta=\pi-\theta_{1}=\pi$ - $\arctan \left(B_{1} / A_{1}\right)$.

The relation curves of the power factor to the control angle $\alpha$ with different values of $K$ are shown in Fig. 5(f). It can be found that as the angle $\alpha$ increases from $90^{\circ}$ to $180^{\circ}$, the power factor remains close-to-unity and increase slightly. When $K$ increases, the power factor decreases.

\section{SimULATION VERIFICATION}

A prototype of the proposed $L C L-T$ resonant converter is simulated with an operation frequency of $25 \mathrm{kHz}$ and a rated output power of 20W. The simulation is carried out by PSIM. The input is $v_{s}=48 \sin \left(\omega_{1} t\right) V$ with an angular frequency $\omega_{1}=50000 \pi \mathrm{rad} / \mathrm{s}$, the MOSFET switches are IRF530N with a $100 \mathrm{~m} \Omega$ on-state resistance, the inductance of $L_{1}$ and $L_{2}$ is $42.5 \mu \mathrm{H}, C$ is a resonant capacitor with $0.94 \mu \mathrm{F}$ and the rated load is $R_{o}=4.1 \Omega$. The simulation results are shown in Fig. 6 .

The input voltage $v_{s}$ and input current $i_{L 1}$ are demonstrated in Fig. 6(a) and 6(b), respectively. The driving signal of $Q_{1}$ and $Q_{2}$ is shown in Fig. 6(c), the resonant current $i_{L 2}$ and reference waveforms $i_{L L}$ are demonstrated in Fig. 6(d), and the output voltage $U_{o}$ is shown in Fig. 6(e).

It can be found from Fig. 6(a) and 6(b) that both the input voltage $v_{s}$ and current $i_{L 1}$ are sinusoidal and that they have the same phase angle. Fig. 6c shows that the driving signal $V_{g}$ can be derived by an input voltage comparison. The charging angle 


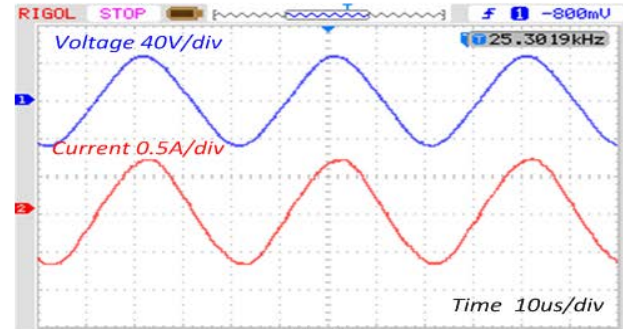

(a)

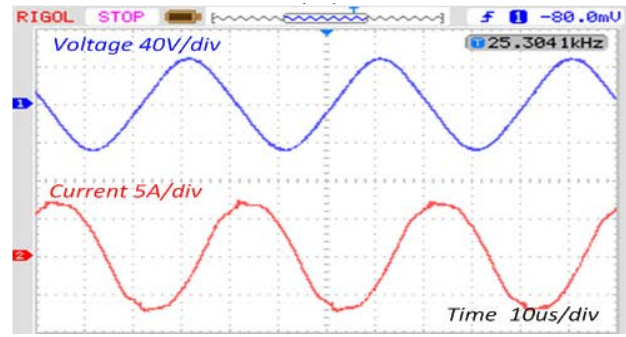

(c)

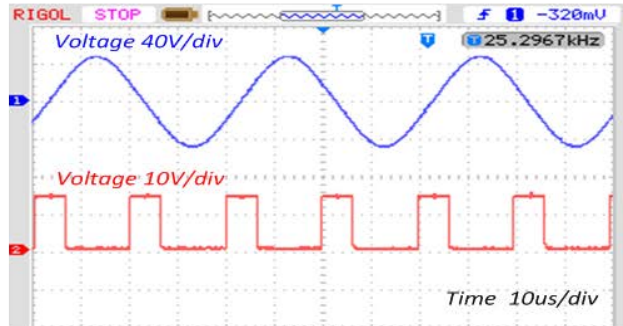

(b)

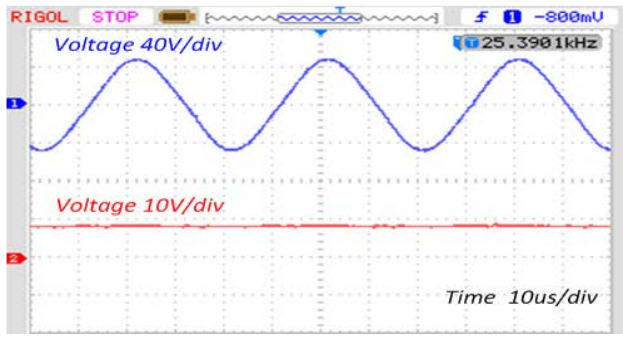

(d)

Fig. 7. The operating waveforms with $48 \sin \left(\omega_{1} t\right) V$ input voltage and charging angle $\alpha=120^{\circ}$ (a) Upper trace: input voltage $v_{s}$, second trace: input current $i_{L 1}$. (b) Upper trace: input voltage $v_{s}$, second trace: the driving signal of $Q_{1}$ and $Q_{2}$. (c) Upper trace: input voltage $v_{s}$, second trace: the inductor current $i_{L 2}$. (d) Upper trace: input voltage $v_{s}$, second trace: the output voltage $U_{o}$.

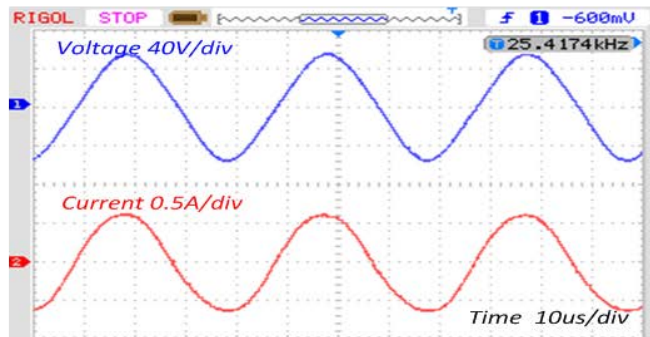

(a)

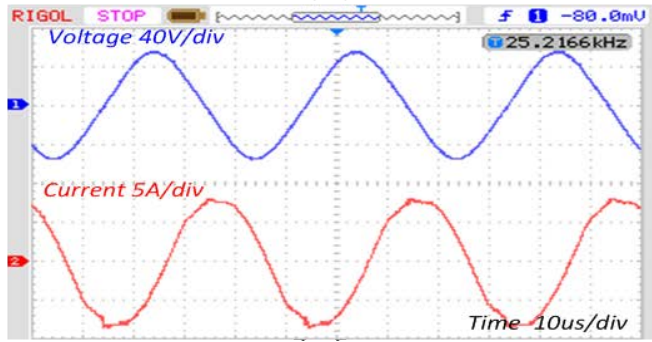

(c)



(b)

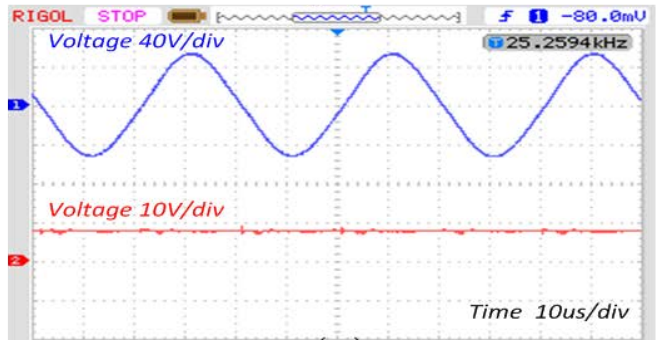

(d)

Fig. 8. The operating waveforms with $55 \sin \left(\omega_{1} t\right) V$ input voltage and charging angle $\alpha=110^{o}$ (a) Upper trace: input voltage $v_{s}$, second trace: input current $i_{L 1}$. (b) Upper trace: input voltage $v_{s}$, second trace: the driving signal of $Q_{1}$ and $Q_{2}$. (c) Upper trace: input voltage $v_{s}$, second trace: the inductor current $i_{L 2}$. (d) Upper trace: input voltage $v_{s}$, second trace: the output voltage $U_{o}$.

of the output capacitor $\alpha$ is between $90^{\circ}$ and $180^{\circ}$, which is used to control the output voltage. The inductor current $i_{L 2}$ is slightly ahead of $i_{L L}$. The calculation results of the PSIM simulation are $P F=0.996$ and $T H D=6.2 \%$. The near-to-unity power factor and the low THD further verify the accuracy of the analysis and the excellent performance of the proposed resonant HFAC/DC converter.

\section{EXPERIMENTAL EVALUATION}

A laboratory prototype is designed and tested to validate the characteristics of the proposed HFAC/DC converter. The same parameters are adopted for both the simulation and the experimental evaluations. The voltages are measured by a Probe Master Model 4231, and the current are detected by a Hantek AC/DC Current Clamp CC-65.

The operating waveforms are illustrated in Fig. 7 with a $48 \sin \left(\omega_{1} \mathrm{t}\right) \mathrm{V}$ input voltage and a charging angle of $\alpha=120^{\circ}$. It can be found that the input current $i_{L 1}$ is sinusoidal and that it has a low harmonics distortion. The input voltage $v_{s}$ and $i_{L 1}$ are almost in the same phase, which ensures a near-to-unity power factor. The resonant current $i_{L 2}$ lags behind $v_{s}$ by nearly $90^{\circ}$, 


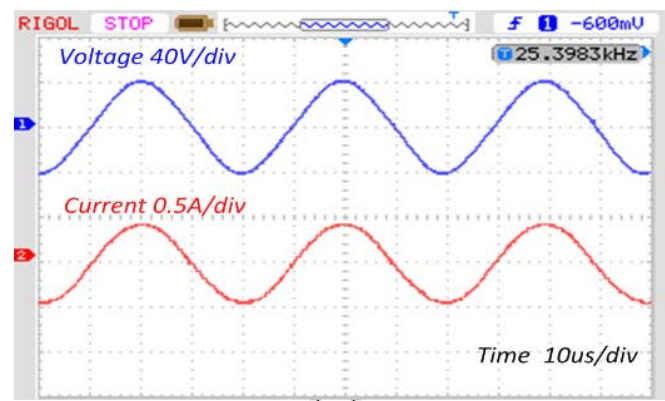

(a)

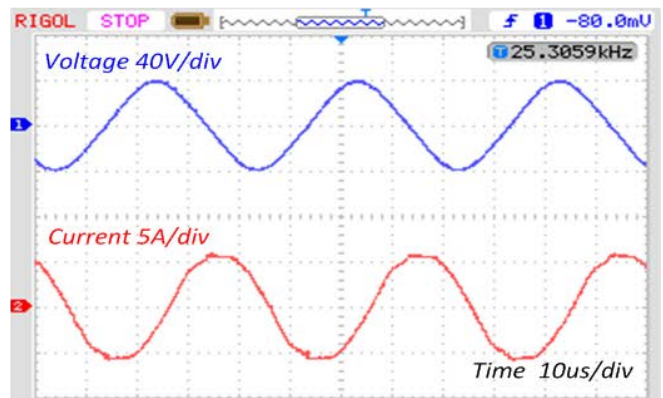

(c)

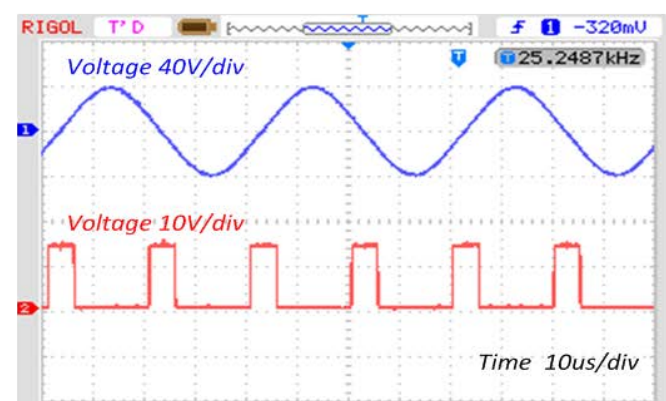

(b)

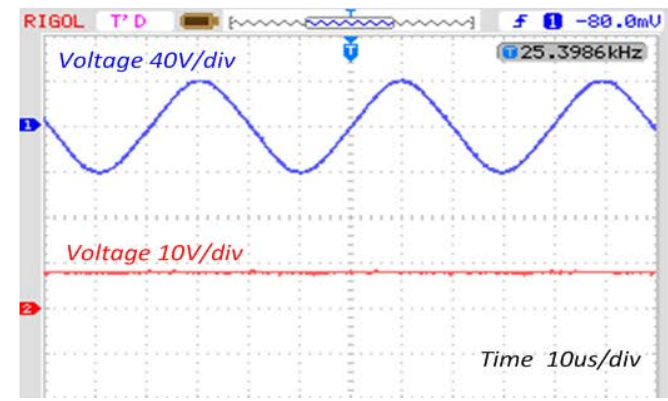

(d)

Fig. 9. The operating waveforms with $40 \sin \left(\omega_{1} t\right) V$ input voltage and charging angle $\alpha=130^{\circ}$. (a) Upper trace: input voltage $v_{s}$, second trace: input current $i_{L 1}$. (b) Upper trace: input voltage $v_{s}$, second trace: the driving signal of $Q_{1}$ and $Q_{2}$. (c) Upper trace: input voltage $v_{s}$, second trace: the inductor current $i_{L 2}$. (d) Upper trace: input voltage $v_{s}$, second trace: the output voltage $U_{o}$.

and the angle $\Phi$ is small. The output voltage $U_{o}$ is $9 \mathrm{~V}$. The experimental waveforms are in good agreement with the simulation results.

A varied input can be found in some applications sourced by batteries, fuel cells, etc., and the proposed converter can regulate the output voltage against input variations. When the experimental converter is fed with varied inputs, the typical waveforms are demonstrated in Fig. 8 and Fig. 9 with different values of the charging angle $\alpha$.

When the input voltage increases to $55 \sin \left(\omega_{1} t\right) V$, as shown in Fig. 8, the output voltage is kept at $9 \mathrm{~V}$ with a charging angle of $\alpha=110^{\circ}$. When the input voltage decreases to $40 \sin \left(\omega_{1} t\right) \mathrm{V}$, as shown in Fig. 9, the output voltage is kept at $9 \mathrm{~V}$ with a charging angle of $\alpha=130^{\circ}$. It can be concluded that the charging angle $\alpha$ can be regulated against input variations. In addition, the capability in the presence of input variations is determined by the operational scope of the charging angle $\alpha$ and the value of $U_{o} / B$. When the charging angle $\alpha$ is operated within the scope of $90^{\circ}$ to $180^{\circ}, U_{o} / B$ is shown in Fig. 5b.

Consequently, the proposed $L C L-T$ resonant converter and control method are effective for the implementation of a high frequency power supply with precise voltage control. Meanwhile, both a low THD and a near-to-unity power factor are achieved over the operational scope.

Meanwhile, it can be found from the experimental results that the resonant current $i_{L 2}$ is about 10A. A larger $i_{L 2}$ results in design difficulty for the inductors, a larger circulation current and increased power losses. It is significant for parameter design to cut down the resonant current.

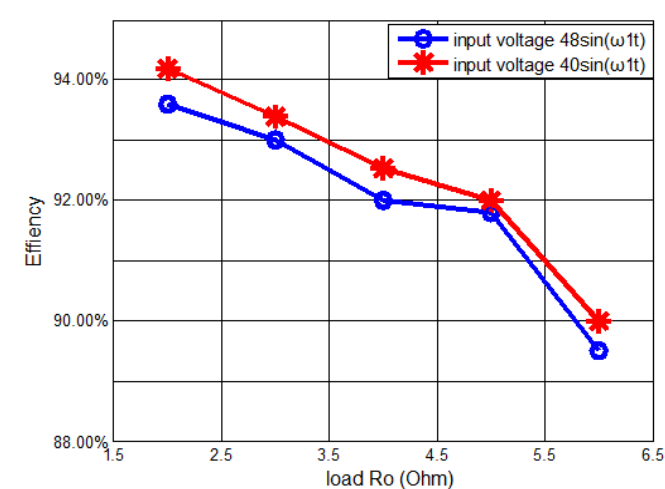

Fig. 10. The curve of efficiency to load and input.

The curve of the efficiency to the load and input is illustrated in Fig.10. The power loss mainly comes from the MOSFETs, the resonant components and the rectifier. In view of the large resonant current, the conducting losses of the MOSFETs are high. It can be found in Fig.10 that the maximum efficiency is close to $94 \%$ at a $2 \Omega$ load; while the minimum efficiency is close to $89 \%$ at a $6 \Omega$ load. As $R_{o}$ increases, the control angle $\alpha$ decreases to make the output stable. Therefore, the increasing conducting angle of the MOSFETs results in the increasing conducting loss and a lowering of the efficiency. It can also be found that the converter efficiency sourced by $48 \sin \left(\omega_{1} t\right) \mathrm{V}$ is lower than that sourced by $40 \sin \left(\omega_{1} t\right) \mathrm{V}$. The resonant current increases along with the increasing of $v_{s}$, and the conducting angle of the MOSFETs increases as well. Hence, the conducting loss becomes large and the conversion efficiency become low along with the ascending input voltage. 
Traditionally, the output voltage of an $L C L-T$ resonant rectifier is regulated by its front-end converter. The resonant tank is designed for optimal parameters with a maximum efficiency [30]. However, the output is uncontrollable for a conventional resonant rectifier since it is incapable of being regulated against input variations. The proposed $L C L-T$ resonant converter provides an effective output control using bidirectional switches. In addition, a wide scope of output regulation can be accomplished by a simple control strategy. In addition, a low distortion and a close-to-unity power factor are achieved for input side.

\section{CONCLUSION}

In order to improve the load performance of HFAC PDS, a controllable HFAC/DC converter is proposed with the modified $L C L-T$ resonant tank. Meanwhile, an easier control method is presented for the proposed converter. The output voltage can be effectively regulated by bidirectional switches. The circuit description, operating principles, and steady-state analysis are examined in depth. A low THD and a near-to-unity power factor are both achieved. A controlled output can be achieved over a wide scope of the charging angle $\alpha$. A simulation schematic and an experimental prototype are implemented with a rated output power of $20 \mathrm{~W}$, a rated frequency of $25 \mathrm{kHz}$, and a rated input peak voltage of $48 \mathrm{~V}$. The experimental results are in good agreement with the theoretical analysis and the simulation results. Consequently, the proposed resonant topology and control method are an effective solution for HFAC/DC conversion.

\section{ACKNOWLEDGMENT}

The authors gratefully acknowledge the financial support of National Natural Science Foundation of China (No.60904078 and No.51177050), the Fundamental Research Funds for the Central Universities of SCUT (No. D2130830), and Technology R\&D Program of Guangzhou (No. 2011J4300024).

\section{REFERENCES}

[1] R. Strzeleki and G. Benysek, Power Electronics in Smart Electrical Energy Networks, Springer, pp.175-201, 2008.

[2] P. Jain and H. Pinheiro, "Hybrid high frequency AC power distribution architecture for telecommunication systems," IEEE Transactions on Aerospace and Electronic Systems, Vol. 35, No. 1, pp. 138-147, Jan. 1999.

[3] J. Liu and K. W. E. Cheng, " $\mu$-based robust controller design of LCLC resonant inverter for high-frequency power distribution system," IET Power Electron., Vol. 6, No. 4, pp. 652-662, April. 2013.

[4] W. Guo and P. K. Jain, “A Power-factor-corrected ac-ac inverter topology using a unified controller for high-frequency power distribution architecture,” IEEE Trans. Ind. Electron., Vol. 51, No. 4, pp. 874-883, Aug. 2004.

[5] B. K. Bose, M.-H. Kin, and M. D. Kankam, "High frequency ac vs dc distribution system for next generation hybrid electric vehicle,” in Proc. IECON, pp. 706-712, 1996.

[6] S. Chakraborty and M. G. Simoes, "Experimental evaluation of active filtering in a single-phase high-frequency ac microgrid," IEEE Trans. Energy Convers., Vol. 24, No. 3, pp. 673-682, Sep. 2009.

[7] J. Liu, K.W.E Cheng, and Y. Ye, “A cascaded multilevel inverter based on switched-capacitor for high-frequency AC power distribution system," IEEE Trans. Power Electron., Vol. 29, No. 8, pp. 4219-4230, Aug. 2014.

[8] S. Luo and I. Batarseh, "A review of distributed power systems part II: High frequency AC distributed power systems,” IEEE Aerosp. Electron. Syst. Mag., Vol. 21, No. 6, pp. 5-14, Jun. 2006.

[9] P. Jain, M. Pahlevaninezhad, S. Pan, and J. Drobnik, “A review of high frequency power distribution systems: for space, telecommunication, and computer applications," IEEE Trans. Power Electron., Vol. 29, No. 8, pp. 3852-3863, Aug. 2014.

[10] P. Jain and M. Tanju, "Improved DC/AC interface inverter for high-frequency space applications," IEEE Trans. Aerosp. Electron. Syst., Vol. 29, No. 4, pp. 1150-1163, Oct. 1993.

[11] J. Liu, K. Wai and E. Cheng, "New power sharing scheme with correlation control for input-parallel-output-seriesbased interleaved resonant inverters," IET Power Electron., Vol. 7, No. 5, pp. 1266-1277, May 2014.

[12] Z. Ye, J. C. W. Lam, and P. C. Sen, "A robust one-cycle controlled full-bridge series-parallel resonant inverter for a high-frequency ac (HFAC) distribution system," IEEE Trans. Power Electron., Vol. 22. No. 6. pp. 2331-2343, Nov. 2007.

[13] Z. Ye, P. K. Jain, and P. C. Sen, “A full-bridge resonant inverter with modified phase-shift modulation for high-frequency ac power distribution systems," IEEE Trans. Ind. Electron., Vol. 54, No. 5, pp. 2831-2845, Oct. 2007.

[14] J. Liu, K. W. E. Cheng, and J. Zeng, “A unified phase-shift modulation for optimized synchronization of parallel resonant inverters in high frequency power system," IEEE Trans. Ind. Electron., Vol. 61, No. 7, pp. 3232-3247, Jul. 2014.

[15] P. Jain and M. C. Tanju, “A unity power factor resonant AC/DC converter for high-frequency space power distribution system,” IEEE Trans. Power Electron., Vol. 12, No. 2, pp. 325-331, Mar. 1997.

[16] J. C. W. Lam and P. K. Jain, “A modified valley fill electronic ballast having a current source resonant inverter with improved line-current total harmonic distortion (THD), high power factor, and low lamp crest factor,” IEEE Trans. Ind. Electron., Vol. 55, No. 3, pp. 1147-1159, Mar. 2008.

[17] J. Qian, F. C. Lee, and T. Yamauchi, “An improved charge pump power factor correction electronic ballast,” IEEE Trans. Power Electron., Vol. 14, No. 6, pp. 1007-1013, Nov. 1999.

[18] M. C. Tanju and P. K. Jain, "High-performance ac/dc converter for high-frequency power distribution systems: analysis, design considerations, and experimental results," IEEE Trans. Power Electron., Vol. 9, No. 3, pp. 275-280, May 1994. 
[19] P. Jain and M. C. Tanju, "A unity power factor resonant AC/DC converter for high-frequency space power distribution system," in Proc. PESC, pp. 3-9, 1994.

[20] F. Musavi, P. K. Jain, and H. Zhang, "A resonant AC/DC converter for high frequency power architecture," in Proc. INTELEC, pp. 497-503, 2002.

[21] C.-M. Lai and R.-C. Lee, "A single-stage AC/DC LLC resonant converter,” in Proc. ICIT, pp. 1386-1390, 2006.

[22] F. Musavi, “A resonant AC/DC converter for high frequency power distribution systems," Master Thesis, Concordia University, Canada, 2001.

[23] M. Qiu, "High frequency AC distributed power system for desktop computer applications," PHD Thesis, Concordia University, Canada, 2004.

[24] Q. Luo, C. Zou, S. Zhi, Hua Yan, L. Zhou, “A multi-channel LED driver based on passive resonant constant current networks," in Proc. the CSEE, Vol. 33, No. 18, pp. 73-79, May 2013.

[25] Q. Luo , S. Zhi , C. Zou, W. Lu, and L. Zhou, “An LED driver with dynamic high-frequency sinusoidal bus voltage regulation for multistring applications,” IEEE Trans. Power Electron., Vol. 29, No. 1, pp. 491-500, Jan. 2014.

[26] H. Pollock, "Simple constant frequency constant current load-resonant power supply under variable load conditions," Electronics Letters, Vol. 33, No. 18, pp. 1505-1506, Aug. 1997.

[27] R. Carbone, H. W. Dommel, R. Langella, and A. Testa, "Analysis and estimation of truncation errors in modeling complex resonant circuits with the EMTP," International Journal of Electrical Power and Energy Systems, Vol. 24, No. 4, pp. 295-304, May 2002.

[28] M. Borage, S. Tiwari, and S. Kotaiah, "Analysis and design of an LCL-T resonant converter as a constant-current power supply," IEEE Trans. Ind. Electron., Vol. 52, No. 6, pp. 1547-1554, Dec. 2005.

[29] M. Borage, S. Tiwari, and S. Kotaiah, "LCL-T resonant converter with clamp diodes: a novel constant-current power supply with inherent constant-voltage limit," IEEE Trans. Ind. Electron., Vol. 54, No. 2, pp. 741-746, Apr. 2007.

[30] I. M. Frivaldský, P. Drgoňa, and P. Špánik, “Experimental analysis and optimization of key parameters of ZVS mode and its application in the proposed LLC converter designed for distributed power system application," International Journal of Electrical Power and Energy Systems, Vol. 47, pp. 448-456, May 2013.



Jun Zeng received her Ph.D. degree in Control Theory and Control Engineering from the South China University of Technology, Guangzhou, China, in 2007. She is an Associate Professor in the School of Electric Power, South China University of Technology. Her current research interests include energy management and intelligence control in distributed generation, and the integration of renewable energy to smart grids.

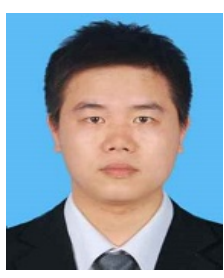

Xuesheng Li was born in Henan, China, in 1991. He received his B.S. degree in Electrical Engineering and Automation from the Henan Polytechnic University, Jiaozuo, China, in 2013. He is presently working toward his M.S. degree in Power Electronics and Drives at South China University of Technology, Guangzhou, China. His current research interests include resonant converters, high frequency power distribution systems, and renewable energy generation.

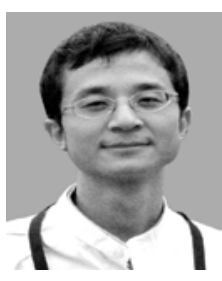

Junfeng Liu received his M.S. degree in Control Engineering from the South China University of Technology, Guangzhou, China, in 2005, and his Ph.D. degree from the Hong Kong Polytechnic University, Kowloon, Hong Kong, China, in 2013. From 2005 to 2008, he was a Development Engineer at Guangdong Nortel Network, Guangzhou, China. In 2014, he joined the South China University of Technology, where he is an Associated Professor in the School of Automation Science and Engineering. His current research interests include power electronics applications, nonlinear control, high frequency power distribution systems, and motion control systems. 